\title{
SMOOTHED PARTICLE HYDRODYNAMICS SIMULATION OF EFFECTIVE THERMAL CONDUCTIVITY IN POROUS MEDIA OF VARIOUS PORE STRUCTURES
}

\author{
Fangming Jiang ${ }^{1, *}$ E Antonio C. M. Sousa $a^{2,3}$ \\ ${ }^{1}$ Guangzhou Institute of Energy Conversion, Chinese Academy of Sciences, No.2 Nengyuan Rd., \\ Tianhe District, Guangzhou 510640, China \\ ${ }^{2}$ Departamento de Engenharia Mecânica, Universidade de Aveiro, Campus Universitário de San- \\ tiago, 3810-193 Aveiro, Portugal \\ ${ }^{3}$ Also at Department of Mechanical Engineering, University of New Brunswick, Fredericton, \\ New Brunswick, E3B 5A3, Canada \\ *Address all correspondence to Fangming Jiang E-mail: fm_jiang2000@yahoo.com \\ Original Manuscript Submitted: 5/4/2009; Final Draft Received: 2/10/2009
}

\begin{abstract}
Heat conduction through a 2-D porous medium layer with complicated cylindrical or quadrangular pore structures is simulated using the smoothed particle hydrodynamics technique. Heat transfer paths are visualized at the micropore level, and the dependence of the effective thermal conductivity on the micropore structure is analyzed. As expected, heat always follows the path of least resistance through the porous structures. Globally, enhanced heat transfer paths tend to form in the porous medium having the smallest circular inclusions. The dependence of the effective thermal conductivity on the micropore structure is found to be closely related to the formation of enhanced heat transfer paths. For the porous medium with dispersed pore phase, the inclusion shape and size and the relative arrangement between inclusions do not have any particular effect on the relation between the effective thermal conductivity and the porosity. This finding is also well predicted by the effective medium theoretical (EMT) model with a flexible factor within the range 4.0-4.5. Owing to the significant effect of the pore-phase distribution, for the porous medium with continuous pore phase, the relation between the effective thermal conductivity and porosity can be predicted using the EMT model only if the flexible factor is taken for a value of 3.5 .
\end{abstract}

KEY WORDS: SPH, porous media, effective thermal conductivity, numerical methods

\section{INTRODUCTION}

The porous medium being considered for this study is a solid matrix permeated by connected or disconnected groups of cylindrical/quadrangular pores filled with a fluid. This type of porous medium is not unusual, and it has been receiving increased attention; particular examples are alumina fiber membranes (Fernando and Chung, 2002), glasses with controlled pores (Gille et al., 2001), and mobile crystalline materials (Wloch et al., 2002).
Porous media have extensive industrial applications, and in addition to those reported in Jiang and Sousa (2006), it should be mentioned that they are widely used in the fuel cell industry. Fuel cells, as energy-conversion devices of high efficiency and low environmental pollution, are widely regarded as the 21 st-century alternative energy system for mobile, stationary, and portable power (Wang, 2004). Fuel cell processes occur primarily in porous media, namely, electrochemical reactions in porous catalyst layers, reactant feeding and product removal through gas 


\section{NOMENCLATURE}

$\begin{array}{ll}C & \text { specific heat capacity }\left(\mathrm{J} \mathrm{Kg}^{-1} \mathrm{~K}^{-1}\right) \\ f & \text { "flexible" factor in the theoretical model } \\ F & \text { any field quantity } \\ h & \text { smoothing length } \\ h_{a} & \text { thickness of the auxiliary layer } \\ h_{s} & \text { thickness of the test sample } \\ k & \text { bulk thermal conductivity } \\ k_{a} & \text { thermal conductivity of the auxiliary medium } \\ k_{e} & \text { effective thermal conductivity } \\ l, L & \text { length } \\ m & \text { mass } \\ n & \text { SPH particle } n \\ N & \text { total SPH particle number } \\ q, \mathbf{q} & \text { heat flux (W m }{ }^{-2} \text { ) } \\ Q & \text { energy flow through the modeling system }(\mathrm{W}) \\ Q_{\text {cold }} & \text { energy flow at the cold wall } \\ Q_{\text {hot }} & \text { energy flow at the hot wall } \\ r, \mathbf{r} & \text { SPH particle position } \\ r_{n} & \text { position of SPH particle } n \\ t & \text { time } \\ T & \text { temperature }\end{array}$

$T_{\text {cold }} \quad$ temperature of the cold wall

$T_{\text {hot }} \quad$ temperature of the hot wall

$w \quad$ smoothing kernel

$x, y \quad$ Cartesian coordinates

$X \quad x$ dimension of the modeling system

$Y \quad y$ dimension of the modeling system

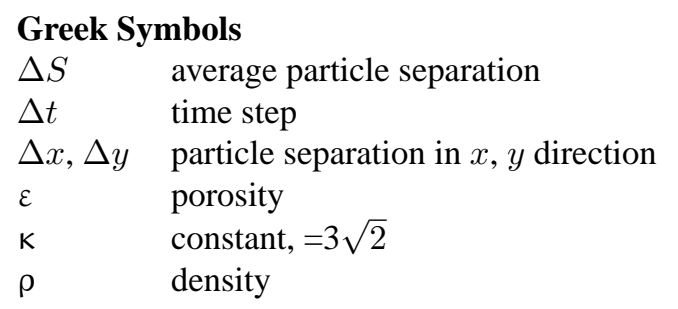

$\begin{array}{ll}\text { Subscripts } \\ 1,2 & \text { index of component } \\ n & \text { index of SPH particle } n \\ j & \text { index of neighboring SPH particle } j \\ n j & \text { certain physical quantity of particle } \\ & n \text { minus that of particle } j\end{array}$

diffusion layers, transport of electrons in porous electrodes, and migration of ions (or protons) in ionomercontaining membranes.

Thermal modeling of physical processes occurring in a porous medium usually requires the predetermination of the effective thermal conductivity $\left(k_{e}\right.$, often referred to as apparent thermal conductivity) of the medium. This parameter is a phenomenological quantity based on a macroscopic equivalence concept, which lumps the detailed features of the microscopic component configurations inside the medium. However, pore-scale numerical simulations can reveal these underlying microscopic features and play an important role in the determination of the correlation between the microscopic component configurations and the macroscopic effective thermal conductivity of the medium. A relatively novel numerical technique - the meshless Lagrangian smoothed particle hydrodynamics (SPH) method-was found to be well suited to modeling thermal processes in porous media when pore-level modeling is required (Jiang and Sousa, 2006).

The present work is a continuation of the work by Jiang and Sousa (2006), and it has a twofold purpose: first, the pore-scale SPH model established in Jiang and Sousa (2006) will be further developed to model the heat conduction in porous media with more involved micropore structures; and second, this enhanced model will be used to predict the effective thermal conductivity in 2-D porous medium layers with different cylindrical or quadrangular pore structures and to explore the dependence of $k_{e}$ on the pore structures.

To facilitate the discussion involved in the present study, the pore phase is defined to be the phase with lower thermal conductivity, and the volume fraction of the pore phase is referred to as the porosity. Only heat conduction will be considered as natural convection of the fluid in the pores is neglected because of the small dimension of the pores and the moderately low temperature differences; that is, the pore Rayleigh number is smaller than the critical Rayleigh number, and it has a typical value less than 0.1. This article is organized as follows: various two-dimensional porous systems are constructed and 
presented in Section 2; Section 3 briefly introduces the pore-scale SPH model; simulation results along with relevant discussion are presented in detail in Section 4; an error estimate to evaluate the reliability of the results is reported in Section 5; and in the last section, the main findings and conclusions are summarized.

\section{VARIOUS MICROPORE STRUCTURES}

The 2-D porous microstructures considered encompass four different types, as depicted in Fig. 1. Circular or square inclusions (pores) with a known bulk thermal conductivity are randomly placed in the solid matrix to form a 2-D porous medium. If the placement of the inclusions is controlled to make them isolated from each other, pore structures type I and II are formed; pore structure type III is formed by dividing the porous medium into regularly distributed squared subdomains and randomly specifying them as a particular component; pore structure type IV is constructed by allowing unlimited overlap between the circular inclusions. For the porous media with the same pore structure type, one porous system differs from another because they have either a different number of inclusions or inclusions of different size. In terms of the pore phase specification rule described in the preceding

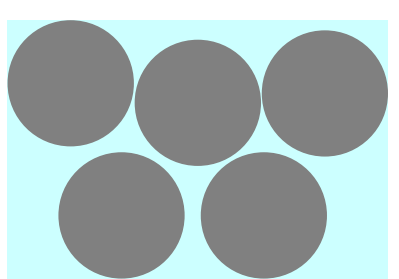

(I)

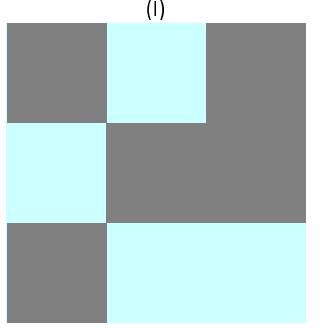

(III)

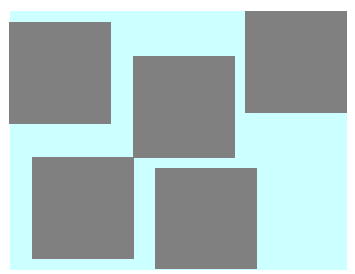

(II)

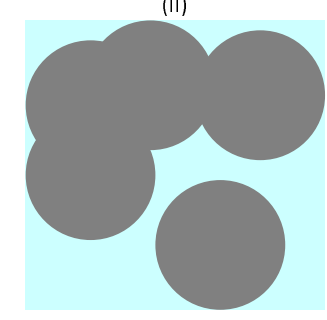

(IV)
FIG. 1: Typical porous microstructures: (I) randomly embedded circles are isolated from each other; (II) randomly embedded squares are isolated from each other; (III) regularly distributed square subdomains are randomly specified to be a particular component; (IV) randomly embedded circles are permitted unlimited overlapping; voids represent a different component section, by exchanging the bulk thermal conductivity between the embedded and the matrix components, the pore phase is thus changed. For porous media of pore structure types I, II, or IV, by adjusting the number of inclusions, it gives a different porosity; for pore structure type III, different porosities can be obtained if different randomness numbers are used in the component specification. The relation between the effective thermal conductivity, $k_{e}$, and porosity can be better understood by analyzing it for different scenarios, namely, the dependence of $k_{e}$ on inclusion shape/size, relative arrangement between inclusions, and the pore phase designation.

\section{NUMERICAL STRATEGY}

The numerical modeling strategy is fully reported in Jiang and Sousa (2006); therefore, here, for the sake of completeness, only a brief description will be given.

\subsection{Theoretical Measurement System of $\boldsymbol{k}_{e}$}

A theoretical measurement system was devised for the determination of the effective thermal conductivity in heterogeneous multicomponent materials; it comprises a rectangular $\left(X \times h_{s}\right)$ piece of a sample sandwiched by two auxiliary layers (of thickness $h_{a}$ each) with two adiabatic sides and the two other sides held at constant temperatures, $T_{\text {hot }}$ and $T_{\text {cold }}$, respectively, as depicted in Fig. 2

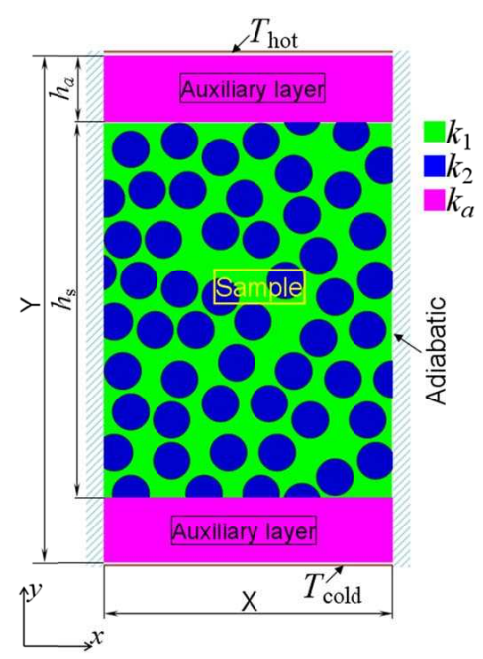

FIG. 2: Theoretical "measurement" system for the determination of the effective thermal conductivity. The background of the plot depicts the component spatial distribution; different colors denote different components 
(only the pore structure type I is illustrated herein). The bulk thermal conductivity of the matrix and the embedded component are $k_{1}$ and $k_{2}$, respectively. The auxiliary layers are used to assess whether steady state has been reached; in these regions of constant thermal conductivity, energy balances are calculated to evaluate the transient development. The thermal conductivity of the auxiliary material $\left(k_{a}\right)$ is much larger than $k_{i}(i=1,2)$, that is, $k_{a} \gg k_{i}$, which means the thermal resistance in the auxiliary medium is negligible in comparison to that in the sample, and thus the introduction of the auxiliary medium has little influence on the accuracy of the $k_{e}$ determination.

The SPH calculation is performed for the transient development of the thermal problem under study up to its steady state. For the present study, the initial temperature is assumed to follow a linear distribution along the $y$ direction. The heat conduction within the test sample (including the auxiliary layers) is governed by the following parabolic partial differential equation:

$$
\begin{aligned}
& C(x, y) \frac{\partial T}{\partial t}=\frac{1}{\rho(x, y)}\left[\frac{\partial}{\partial x}\left(k(x, y) \frac{\partial T}{\partial x}\right)\right. \\
& \left.+\frac{\partial}{\partial y}\left(k(x, y) \frac{\partial T}{\partial y}\right)\right]=\frac{1}{\rho(x, y)} \nabla \cdot(k(x, y) \nabla T)
\end{aligned}
$$

The steady state can be verified by performing energy balances at the two constant-temperature boundaries. If the relative difference between the energy flows through the cold wall $\left(Q_{\text {cold }}\right)$ and hot wall $\left(Q_{\text {hot }}\right)$ is less than $1.0 \%$ for the same instant of time, and the relative variation of the energy flow, $Q_{\text {hot }}$, between two neighboring time instants $(t$ and $t+\Delta t)$ is less than $10^{-5}$, the steady state is considered to have been achieved. Then the arithmetical mean of $Q_{\text {cold }}$ and $Q_{\text {hot }}$ is taken to be the final steady state energy flow, $Q$, through the system, and the effective thermal conductivity $k_{e}$ of the sample is determined as follows:

$$
k_{e}=\frac{h_{s}}{\frac{1 \cdot X\left(T_{\mathrm{hot}}-T_{\mathrm{cold}}\right)}{Q}-\frac{2 h_{a}}{k_{a}}}
$$

\subsection{SPH Formulation and Methodology}

The concept behind the SPH is based essentially on an interpolation technique. In the implementation of the SPH method to solve heat conduction problems, the thermal quantity at time $t$ is represented by a collection of $N$ particles located at position $\mathbf{r}_{n}, n=1,2, \ldots, N$ (bold text designates a vector). The smoothed value of any field quantity $F(\mathbf{r}, t)$ at a space point $\mathbf{r}$ and at a time $t$ is a weighted sum of all contributions from the neighboring particles, namely,

$$
\langle F(\mathbf{r}, t)\rangle=\sum_{j=1}^{N} \frac{m_{j}}{\rho_{j}} F\left(\mathbf{r}_{j}, t\right) w\left(\left|\mathbf{r}-\mathbf{r}_{j}\right|, h\right)
$$

The commonly used symmetric SPH formulation for the first-order derivative of $F(\mathbf{r}, t)$ is written as

$$
\begin{aligned}
& \langle\nabla F(\mathbf{r}, t)\rangle=\sum_{j=1}^{N} \frac{m_{j}}{\rho_{j}}\left[F\left(\mathbf{r}_{j}, t\right)-F(\mathbf{r}, t)\right] \\
& \times \nabla w\left(\left|\mathbf{r}-\mathbf{r}_{j}\right|, h\right)
\end{aligned}
$$

Cleary and Monaghan (1999) gave the SPH form of the second-order diffusion term in Eq. (1) as

$$
\frac{1}{\rho} \nabla \cdot(k \nabla T)=\sum_{j} \frac{4 m_{j}}{\rho_{n} \rho_{j}} \frac{k_{n} k_{j}}{k_{n}+k_{j}} T_{n j} \frac{1}{\left|\mathbf{r}_{n j}\right|} \frac{\partial w_{n j}}{\partial r_{n}}
$$

By substituting Eq. (5) into Eq. (1), the SPH form of the heat conduction equation yields

$$
C(x, y) \frac{\partial T}{\partial t}=\sum_{j} \frac{4 m_{j}}{\rho_{n} \rho_{j}} \frac{k_{n} k_{j}}{k_{n}+k_{j}} T_{n j} \frac{1}{\left|\mathbf{r}_{n j}\right|} \frac{\partial w_{n j}}{\partial r_{n}}
$$

In terms of Eq. (4), the heat flux vector (q) can be calculated with the following SPH formulation:

$$
\mathbf{q}=-k \nabla T=\sum_{j} \frac{2 m_{j}}{\rho_{j}} \frac{k_{n} k_{j}}{k_{n}+k_{j}} T_{n j} \frac{\mathbf{r}_{n j}}{\left|\mathbf{r}_{n j}\right|} \frac{\partial w_{n j}}{\partial r_{n}}
$$

The double-subscripted variables in Eqs. (5)-(7) are defined as $\mathbf{r}_{n j}=\mathbf{r}_{n}-\mathbf{r}_{j}, w_{n j}=w\left(\left|\mathbf{r}_{n}-\mathbf{r}_{j}\right|, h\right)$ and $T_{n j}=$ $T\left(\mathbf{r}_{n}, t\right)-T\left(\mathbf{r}_{j}, t\right)$. A direct numerical integration algorithm is used to perform the integration of Eq. (6). The time step $\Delta t$ is determined by the following equation given by Cleary and Monaghan (1999) and Cleary (1998):

$$
\Delta t=\min _{\mathrm{n}}\left(\frac{0.1 \rho_{n} C_{n} h^{2}}{k_{n}}\right)
$$

In the present SPH implementation, the high-order 3splines quintic smoothing kernel (Jiang and Sousa, 2006) is used, which, for 2-D problems, is formulated as

$$
w_{n j}=\frac{7}{478 \pi h^{2}}\left\{\begin{array}{l}
(3.0-s)^{5}-6.0(2.0-s)^{5} \\
+15.0(1.0-s)^{5} \quad \text { if } 0 \leq s<1.0 \\
(3.0-s)^{5}-6.0(2.0-s)^{5} \\
\text { if } 1.0 \leq s<2.0 \\
(3.0-s)^{5} \quad \text { if } 2.0 \leq s<3.0 \\
0 \text { if } \quad s \geq 3.0
\end{array}\right.
$$


SPH particles are uniformly distributed within the calculation domain. The separation distance between neighboring particles is $\Delta x$ in the $x$ direction and $\Delta y$ in the $y$ direction; and $\Delta x=\Delta y=\Delta S$. The smoothing length $h$ is specified to be $\sqrt{2} \Delta S$. Thus the interpolation of the field quantity with respect to one SPH particle extends to a circular region with a radius of $\kappa h(\kappa=3 \sqrt{2})$.

The constant-temperature wall and adiabatic boundary are properly handled by employing the strategy of ghost boundary particles, which has been described in great detail in Jiang and Sousa (2006) and thus is omitted herein.

\section{RESULTS AND DISCUSSION}

The simulated domain is a rectangle of dimensions $X \times Y$ $\left(Y=h_{s}+2 h_{a}\right)$. In the present study, $X=80 \mathrm{~mm}, Y=$ $90 \mathrm{~mm}$, and $h_{a}=5.5 \mathrm{~mm}$, and thus $h_{s}=79 \mathrm{~mm}$; constant temperatures at the two horizontal boundaries are specified to be $298 \mathrm{~K}$ ( $T_{\text {hot }}$ ) and $278 \mathrm{~K}$ ( $T_{\text {cold }}$ ), respectively; If the pore phase is referred to the inclusions, then the values of the thermal conductivities are $k_{1}=200 \mathrm{~W} / \mathrm{m} \mathrm{K}$ and $k_{2}=10 \mathrm{~W} / \mathrm{m} \mathrm{K}$, respectively; whereas the values of $k_{1}=10 \mathrm{~W} / \mathrm{m} \mathrm{K}$ and $k_{2}=200 \mathrm{~W} / \mathrm{m} \mathrm{K}$, respectively, are specified when the pore phase is the matrix component; $k_{a}$ is specified to be 20 times the bulk thermal conductivity of the non-pore phase, and $\Delta S=1 \mathrm{~mm}$. The specific heat capacity and the density have no influence on the final steady state temperature field; but because the steady state was obtained through a transient process, for convenience, it used the artificial data $\rho_{1}=\rho_{2}=2000 \mathrm{~kg} / \mathrm{m}^{3}$, $C_{1}=C_{2}=1000 \mathrm{~J} / \mathrm{kg} \mathrm{K}$; while $\rho_{a}=5 \rho_{1}, C_{a}=4 C_{1}$, which facilitates the calculation of the time step $\Delta t$ [Eq. (8)] by not requiring the introduction of an artificially large conductivity for the auxiliary medium. The basic SPH numerical elements are $1 \times 1 \mathrm{~mm}$ minisquares each with the SPH particle placed at its center. The SPH particles located on the region of the inclusions are specified to be the component different from the matrix component. The porosity of the porous system is calculated using the following relation:

$$
\varepsilon=\frac{\text { number of the pore phase particles }}{\text { total number of SPH particles in the sample }}
$$

\subsection{Microscopic Visualization of Heat Transfer Paths}

The porous microstructures influence the heat transfer paths, which essentially dictate the dependence of $k_{e}$ on the pore structures. By depicting the heat flux vectors, pore-level heat transfer paths can be visualized.
For porous systems of microstructures type I and II, the identification of the continuous and dispersed phases is evident. If the non-pore phase (of larger bulk thermal conductivity) is continuous, the formation of enhanced heat transfer paths through the medium is facilitated, as shown in Figs. 3 and 4.

For porous systems of microstructures type III and IV, the continuous phase is the dominant one. If the porosity is low, the non-pore phase is continuous, and enhanced heat transfer paths through the whole medium are easily established; if the porosity is high, the pore phase is continuous, and globally enhanced heat transfer paths have limited reach. Pore-scale heat flux vectors for these types of porous media are reported in Figs. 5 and 6. As illus-

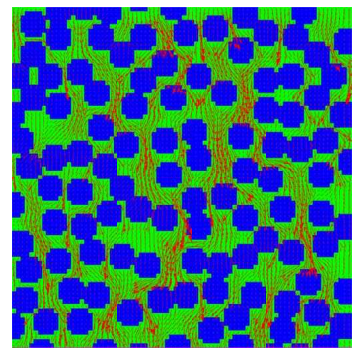

(a)

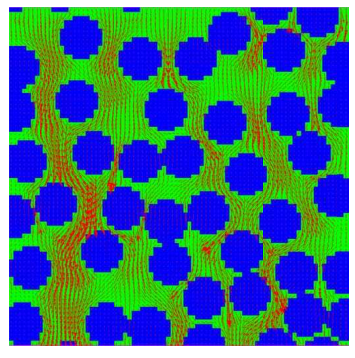

(b)
FIG. 3: Heat flux vectors in the porous system of pore structure type I and with dispersed pore phase: (a) inclusion diameter $6 \mathrm{~mm}, \varepsilon=0.516$; (b) inclusion diameter $10 \mathrm{~mm}, \varepsilon=0.487$ (note that the length scales for the heat flux vectors in the two plots are not the same); the background of the plot depicts the component spatial distribution, where different colors denote different components

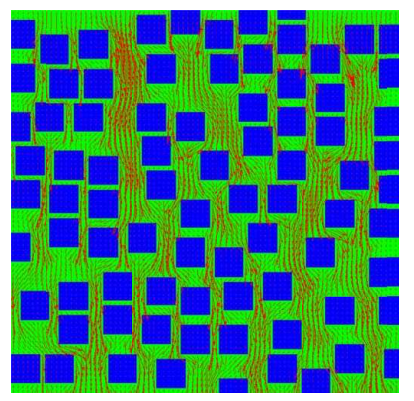

FIG. 4: Heat flux vectors in the porous system of pore structure type II and with dispersed pore phase: $6 \times 6 \mathrm{~mm}$ square inclusions, $\varepsilon=0.44$; the background of the plot depicts the component spatial distribution, where different colors denote different components 


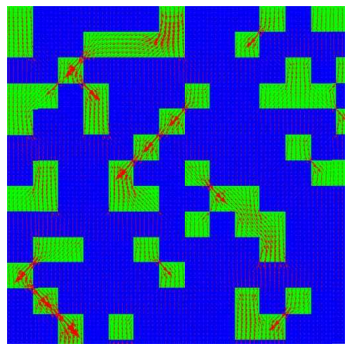

(a)

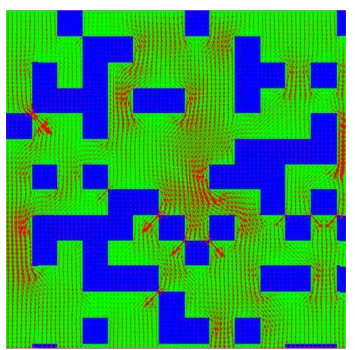

(b)
FIG. 5: Heat flux vectors in the porous system of pore structure type III and with $6 \times 6 \mathrm{~mm}$ subdivided squares: (a) $\varepsilon=0.707$; (b) $\varepsilon=0.315$ (note that the length scales for the heat flux vectors in the two plots are not the same); the background of the plot depicts the component spatial distribution, where different colors denote different components

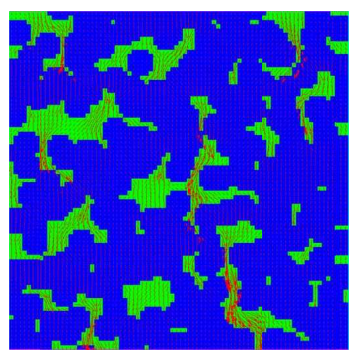

(a)

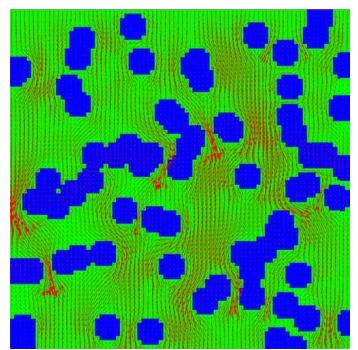

(b)
FIG. 6: Heat flux vectors in the porous system of pore structure type IV and with circular inclusions of $6 \mathrm{~mm}$ diameter: (a) $\varepsilon=0.793$; (b) $\varepsilon=0.297$ (note that the length scales for the heat flux vectors in the two plots are not the same); the background of the plot depicts the component spatial distribution, where different colors denote different components

trated by Fig. 6, in the porous medium of pore structure type IV, owing to the unlimited overlapping between inclusions, the inclusions coalesce and form inclusions with varying shapes and sizes, which further forces the meandering of the enhanced heat transfer paths.

For porous systems of microstructure type I, if the inclusions are specified as the non-pore phase, the pore phase becomes continuous, and the heat has to be transferred mostly through the pore phase. No globally enhanced heat transfer paths are formed, as can be noted in Fig. 7.

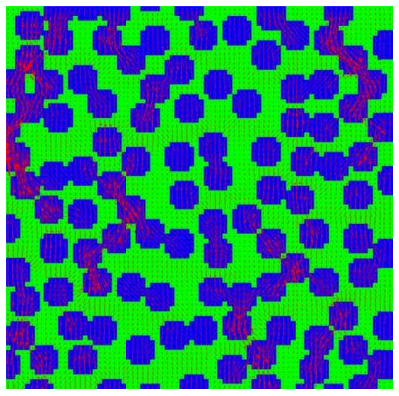

FIG. 7: Heat flux vectors in the porous system of pore structure type I and with continuous pore phase: inclusion diameter $6 \mathrm{~mm}, \varepsilon=0.484$; the background of the plot depicts the component spatial distribution, where different colors denote different components

Close observation of Figs. 3-7 indicates that the heat transfer involved in porous systems of different microstructures does not exhibit substantially different patterns-heat, as expected, always follows the path of least resistance through the porous structures. Even so, some minor differences about the enhanced heat transfer paths in porous systems of different micropore structures is still apparent in Figs. 3-7. Clearly, for porous systems of microstructures type I or II, if the inclusions are specified as the non-pore phase, globally enhanced heat transfer paths through the whole medium cannot form. For porous systems with inclusions as the pore phase, globally enhanced heat transfer paths get established more easily in the systems of microstructure type I than in those of microstructure type IV; similar features occur for systems of microstructure type III, as compared to the systems of microstructure type II. Circular inclusions (pore phase) can smooth the interfaces between pore and nonpore phases, and thus it facilitates the formation of globally enhanced heat transfer paths to form in the porous systems of microstructure type I as compared to the systems of microstructure type II. For the same type porous systems, if the non-pore phase inclusions have larger size, a globally enhanced heat transfer path is generally harder to establish. Most of these observations can be explained intuitively by the resemblance of heat transfer in a porous medium to fluid flow across solid obstacles - the fluid flow is favored if the obstacles have a streamlined shape, smaller size, and/or the obstacles do not hinder the formation of continuous flow pathways.

SPH results allow the visualization of the pore-scale heat transfer details as well as the determination of the ef- 
fective thermal conductivity, $k_{e}$, of the porous systems concerned. The steady state temperature field obtained from the SPH simulation also permits the determination of the overall energy flow, $Q$. By substituting the $Q$ value obtained and other relevant parameters into Eq. (2), the effective thermal conductivity of the porous sample is obtained. For the same type porous systems, a set of simulations with porous systems of different porosity values is performed, from which a relation between $k_{e}$ and porosity can be derived. By comparing the trends of $k_{e}$ to the porosity for porous systems with different microstructures, the effect of the microstructure on $k_{e}$ then can be examined.

\subsection{Effect of the Inclusion Shape on $\boldsymbol{k}_{e}$}

The porous systems for micropore structure type I have inclusions of circular shape; while the pore structure type II consists of square inclusions. The SPH simulated values of $k_{e}$ of these two types of porous systems clearly reveal the effect of inclusion shape on $k_{e}$. Figure 8 displays two sets of $k_{e}$ data: one is from the porous systems of pore structure type I with $6 \mathrm{~mm}$ diameter (D) circular inclusions; and the other is from the porous systems of pore structure type II with $6 \mathrm{~mm}$ side length square inclusions. The deviation between the two sets of data is minor, which indicates that the inclusion shape has little effect on $k_{e}$. Nevertheless, careful examination of Fig. 8 indicates that the values of $k_{e}$ of the porous systems with pore structure type II are, in general, slightly smaller than those of the porous systems with pore structure type I.

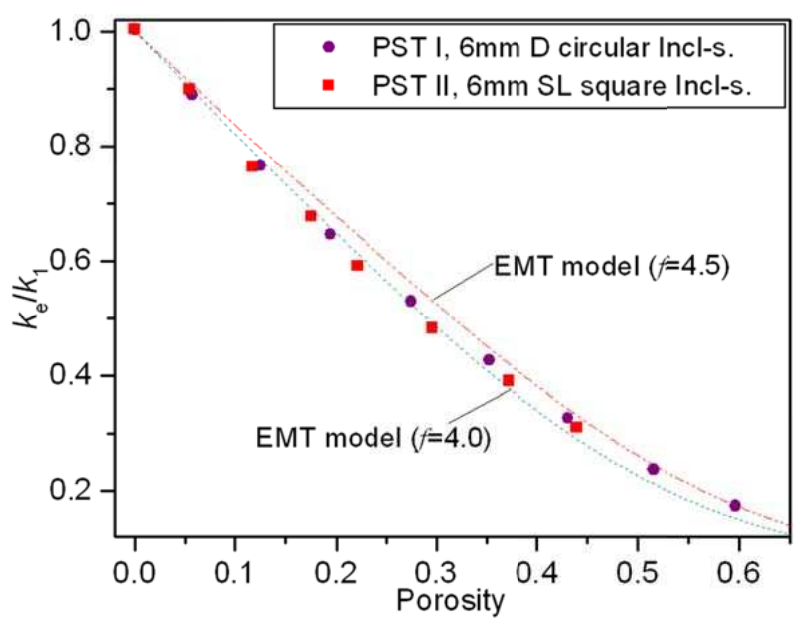

FIG. 8: Effect of inclusions shape on $k_{e}$
This aspect can be explained by the fact that a circular pore phase tends to impose fewer constraints on the globally enhanced heat transfer paths than a square pore phase, as indicated by Figs. 3 and 4 .

The flexible effective medium theoretical (EMT) model (as formulated in Appendix A) introduces an empirical factor $f$ to accommodate the effect of the pore structure on $k_{e}$. From Fig. 8, it is observed the flexible EMT model can predict well the $k_{e}$ of the porous systems with pore structure type I or II when the flexible factor $f$ takes a value in the range 4.0-4.5.

\subsection{Effect of the Inclusion's Relative Arrangement on $\boldsymbol{k}_{e}$}

The inclusion's relative arrangements being considered in the present study to examine their effect on the effective thermal conductivity $k_{e}$ are circular inclusions with either full isolation (pore structure type I) or unlimited overlapping (pore structure type IV) from each other or regularly distributed square subdomains with randomly specified pore phase (pore structure type III). As shown in Fig. 9, the inclusion's relative arrangement has a minor effect on $k_{e}$. As globally enhanced heat transfer paths are easier to form in the porous media of pore structure type I, as shown in Figs. 3, 5 and 6, slightly larger values of $k$ are generally observed for this type of porous medium. Figure 9 clearly indicates that the flexible EMT model can predict well the $k_{e}$ of the porous systems with pore structure types I, III, and IV, when the flexible factor $f$ takes a value within the interval 4.0-4.5.

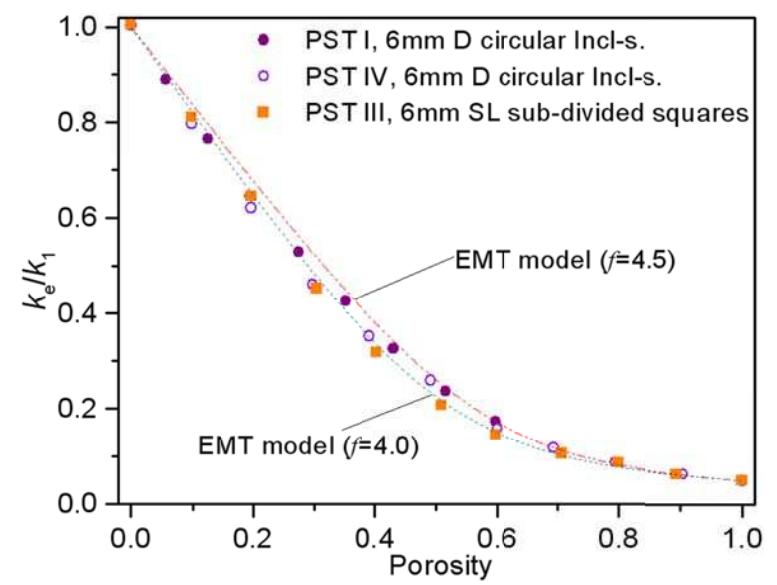

FIG. 9: Effect on $k_{e}$ of the relative arrangement between inclusions 


\subsection{Effect of the Inclusion's Size on $\boldsymbol{k}_{e}$}

Inclusions of larger size tend to further hinder the heat transfer paths in the porous medium, as already reported in Fig. 3. Porous media of pore structure types I and III with different inclusion sizes were selected to investigate the dependence of $k_{e}$ on the size of the inclusion. From the results shown in Fig. 10, for the same type of porous medium, slightly larger values of the effective thermal conductivity can be observed if the size of the inclusion is smaller. Even so, the inclusion size still has no significant effect on the effective thermal conductivity $k_{e}$. The flexible EMT model with a flexible factor $f$ within the interval $4.0-4.5$ is found to be able to predict well the values of $k_{e}$ of the porous systems of pore structure types I and III.

\subsection{Effect of Pore-Phase Designation on $\boldsymbol{k}_{e}$}

Pore-phase designation directly dictates the heat transfer paths. If the pore phase is dispersed, globally enhanced heat transfer paths are easy to form, as depicted by Fig. 3; however, if the pore phase is continuous, no enhanced heat transfer paths can pass through the porous medium (Fig. 7). The effect of the pore-phase designation on $k_{e}$ is reported in Fig. 11. The pore-phase designation brings significant effect on the effective thermal conductivity, $k_{e}$. Once again, the values of $k_{e}$ of porous media with dispersed pore phases can be predicted well by the EMT model with the value of $f$ taken within the interval 4.04.5; for porous media with continuous pore phases, the values of $k_{e}$ can only be predicted with the EMT model using a value of $f$ equal to 3.5. The Maxwell-Hamilton

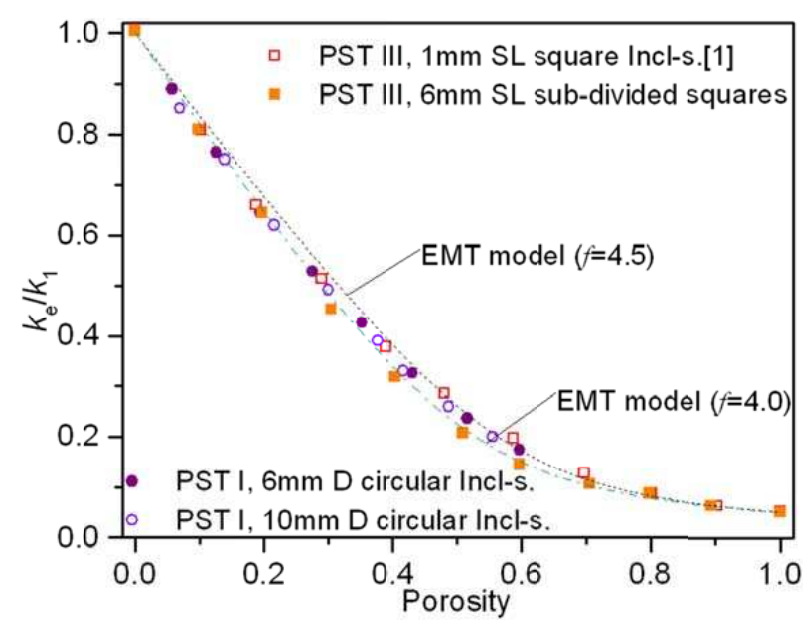

FIG. 10: Effect of inclusion size on $k_{e}$

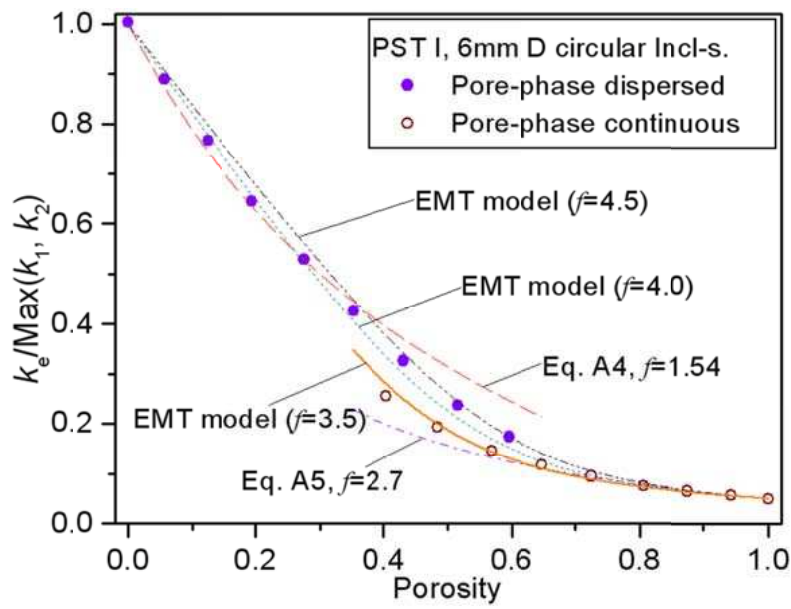

FIG. 11: Effect of the pore phase designation on $k_{e}$

model, as defined in Appendix A, cannot predict the effective thermal conductivity no matter if the pore phase is dispersed or continuous, as shown in Fig. 11.

\section{ERROR ESTIMATE}

To assess the numerical error, two simplified porous system cross sections are considered, as displayed in Fig. 12, namely, a square of side length equal to $6 \mathrm{~mm}$ and a circular inclusion with a $6 \mathrm{~mm}$ diameter, both centered in a squared domain of side length $L$. In the SPH implementation, the inclusions in the porous media are numerically approximated by a number of $1 \times 1 \mathrm{~mm}$ square elements (SPH particles). Obviously, because of its finite resolution, the circular inclusions cannot be accurately represented by the SPH particles, as illustrated in Fig. 12(b),

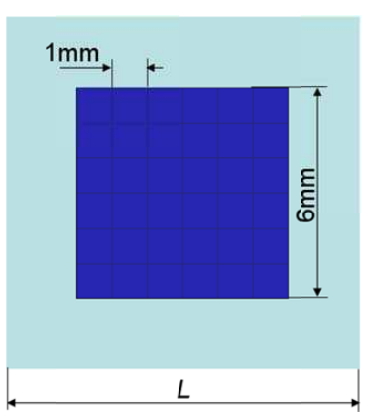

(a)

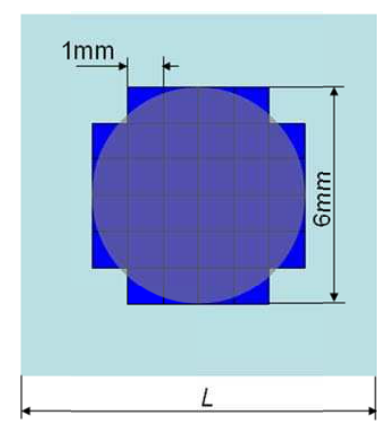

(b)
FIG. 12: Simplified porous systems used in the error estimate: (a) square and (b) circular inclusions 
TABLE 1: Error estimate

\begin{tabular}{|c|c|c|c|c|c|c|c|c|}
\hline & \multicolumn{4}{|l|}{$\begin{array}{l}\text { Square inclusion, 6.0 mm } \\
\text { side length (Fig. 12a) }\end{array}$} & \multicolumn{4}{c|}{$\begin{array}{l}\text { Circular inclusion, 6.0 mm } \\
\text { diameter (Fig. 12b) }\end{array}$} \\
\hline$L(\mathrm{~mm})$ & 80.0 & 30.0 & 20.0 & 10.0 & 80.0 & 30.0 & 20.0 & 10.0 \\
\hline Error $(\%)$ & 0.28 & 3.19 & 6.79 & 17.91 & 0.15 & 2.55 & 5.65 & 16.03 \\
\hline
\end{tabular}

introducing errors into the final $k_{e}$ data. These errors, combined with a classical numerical error analysis, can be generally evaluated by a grid-dependence test. In the following numerical error evaluation, the dimension of the numerical element is fixed equal to $1 \times 1 \mathrm{~mm}$, while the size $(L)$ of the whole domain is varied.

Analytical solutions of $k_{e}$ for these two simplified porous systems are presented in Appendix B. Comparisons between the analytical and numerical results were made, and the errors are summarized in Table 1 . The numerical error is greatly dependent on the geometrical dimension of the porous system. For most of the porous systems of interest in the present work, which have geometrical dimensions of approximately $80 \times 80 \mathrm{~mm}$ and inclusions of characteristic size 6 or $10 \mathrm{~mm}$, the numerical error should be within $1 \%$.

\section{CONCLUSIONS}

The present work is an extension of the study of Jiang and Sousa (2006), and it considers 2-D layers of porous media with relatively complicated cylindrical or quadrangular pore structures. SPH simulations of heat conduction through these porous media visualize the heat transfer paths at the micropore level and reveal the dependence of the effective thermal conductivity on the micropore structure. As expected, the results corroborate that heat always follows the path of least resistance through the porous structures. It is found that globally enhanced heat transfer paths are facilitated for porous media with smaller circular inclusions. If the pore phase is continuous, no globally enhanced heat transfer paths through the porous medium are established. The dependence of the effective thermal conductivity on the micropore structure is found to be closely associated with the formation of enhanced heat transfer paths. Generally, if the pore structure of a medium is favorable to the formation of globally enhanced heat transfer paths, then the effective thermal conductivity will take higher values. For the porous medium with dispersed pore phase, it is found that the relation between the effective thermal conductivity and the porosity has only a minor dependence on the inclusion shape/size and on the relative arrangement between inclusions. The EMT model with a flexible factor within the interval 4.04.5 can well predict the effective thermal conductivity of these types of porous media. The pore-phase designation is found to have significant effect on the effective thermal conductivity versus porosity relation. For the porous media with continuous pore phases, this relation can be predicted by the EMT model with a flexible factor equal to 3.5 .

\section{ACKNOWLEDGEMENTS}

Partial financial support received from the Foundation for Science and Technology, Portugal, research grant POCTI/EME/59728/2004, and the Natural Sciences and Engineering Research Council of Canada, Discovery Grant 12875 (A.C.M.S.), is acknowledged; the postdoctoral fellowship SFRH/BPD/20273/2004 (F.J.) is also acknowledged.

\section{REFERENCES}

Carson, J. K., Lovatt, S. J., Tanner, D. J., and Cleland, A. C., Predicting the effective thermal conductivity of unfrozen porous foods, J. Food Eng., vol. 75, pp. 297-307, 2006.

Cleary, P. W., Modeling confined multi-material heat and mass flows using SPH, Appl. Math. Modell., vol. 22, pp. 981-993, 1998.

Cleary, P. W. and Monaghan, J. J., Conduction modeling using smoothed particle hydrodynamics, J. Comput. Phys., vol. 148, pp. 227-264, 1999.

Fernando, J. A. and Chung, D. D. L., Pore structure and permeability of an alumina fiber filter membrane for hot gas filtration, J. Porous Mater., vol. 9, pp. 211-219, 2002.

Fu, X., Viskanta, R., and Gore, J. P., Prediction of effective thermal conductivity of cellular ceramics, Int. Commun. Heat Mass Transfer, vol. 25, pp. 151-160, 1998.

Gille, W., Enke, D., and Janowski, F., Stereological macropore analysis of a controlled pore glass by use of small-angle scattering, J. Porous Mater., vol. 8, pp. 179-191, 2001.

Jiang, F. and Sousa, A. C. M., Effective thermal conductivity of heterogeneous multi-component materials: An SPH implementation, Heat Mass Transfer, vol. 43, pp. 479-491, 2006. 
Wang, C. Y., Fundamental models for fuel cell engineering, Chem. Rev., vol. 104, pp. 4727-4766, 2004.

Wloch, J., Rozwadowski, M., Lezanska, M., and Erdmann, K., Analysis of the pore structure of the MCM-41 materials, Appl. Surf. Sci., vol. 191, pp. 368-374, 2002.

\section{APPENDIX A. THEORETICAL/SEMIEMPIRICAL MODELS OF $\boldsymbol{K}_{E}$}

Models used for the assessment of the SPH modeling results for $k_{e}$ of two-component porous media are succinctly reviewed in this appendix. Further information on these theoretical/semiempirical models can be found in Carson et al. (2006):

Parallel Model:

$$
k_{e}=\frac{1}{\frac{\varepsilon_{1}}{k_{1}}+\frac{\varepsilon_{2}}{k_{2}}}
$$

Series Model:

$$
k_{e}=k_{1} \varepsilon_{1}+k_{2} \varepsilon_{2}
$$

Flexible EMT Model:

$$
\begin{aligned}
& k_{e}=\frac{1}{f-2}\left\{\left(\frac{f}{2} \varepsilon_{2}-1\right) k_{2}+\left(\frac{f}{2} \varepsilon_{1}-1\right) k_{1}\right. \\
& \left.+\sqrt{\left[\left(\frac{f}{2} \varepsilon_{2}-1\right) k_{2}+\left(\frac{f}{2} \varepsilon_{1}-1\right) k_{1}\right]^{2}+(2 f-4) k_{1} k_{2}}\right\}
\end{aligned}
$$

Maxwell-Hamilton Model (Phase 1 Continuous):

$$
k_{e}=k_{1} \frac{(f-1) k_{1}+k_{2}-(f-1)\left(k_{1}-k_{2}\right) \varepsilon_{2}}{(f-1) k_{1}+k_{2}+\left(k_{1}-k_{2}\right) \varepsilon_{2}}
$$

Maxwell-Hamilton Model (Phase 2 Continuous):

$$
k_{e}=k_{2} \frac{(f-1) k_{2}+k_{1}+(f-1)\left(k_{1}-k_{2}\right)\left(1-\varepsilon_{2}\right)}{(f-1) k_{2}+k_{1}-\left(k_{1}-k_{2}\right)\left(1-\varepsilon_{2}\right)}
$$

\section{APPENDIX B. TWO ANALYTICAL EXPRESSIONS FOR $\boldsymbol{K}_{E}$}

The value of $k_{e}$ for any porous system can be derived using the thermal-circuit method (Fu el al., 1998). Analytical expressions of $k_{e}$ for two idealized porous systems (Figs. 13 and 14) that were employed to estimate the error of the SPH calculated data are presented in this Appendix.

In terms of Eqs. (A1) and (A2), the effective thermal conductivity for the porous system depicted in Fig. 13 can be expressed as

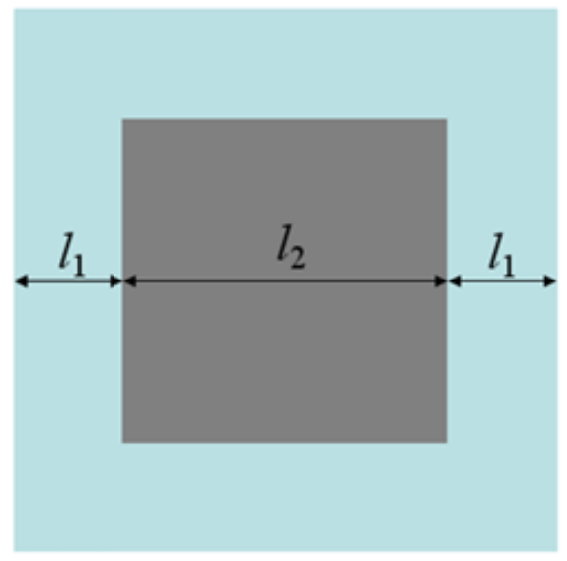

FIG. 13: A 2-D idealized porous system: a square domain with a centered square inclusion (phase 2)

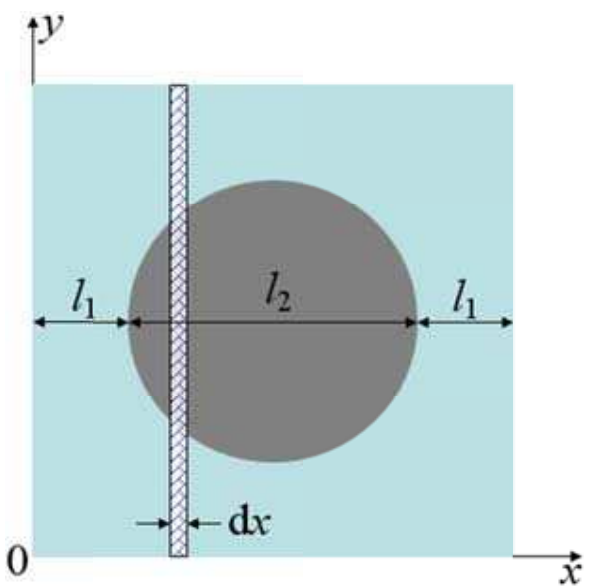

FIG. 14: Same as Fig. A1, but for a circular inclusion

$$
k_{e}=\frac{1}{\frac{l_{2}}{2 l_{1} k_{1}+l_{2} k_{2}}+\frac{2 l_{1}}{\left(l_{2}+2 l_{1}\right) k_{1}}}
$$

The effective thermal conductivity for the porous system illustrated in Fig. 14 is formulated as follows:

$$
\begin{aligned}
& k_{e}=1 /\left\{\int_{l_{1}}^{l_{1}+l_{2}}(d x) /\left(k_{1}\left[\left(l_{2}+2 l_{1}\right)-\sqrt{l_{2}^{2}-\left(l_{2}+2 l_{1}-2 x\right)^{2}}\right]\right.\right. \\
& \left.\left.+k_{2} \sqrt{l_{2}^{2}-\left(l_{2}+2 l_{1}-2 x\right)^{2}}\right)+\frac{2 l_{1}}{\left(l_{2}+2 l_{1}\right) k_{1}}\right\}
\end{aligned}
$$

